\title{
Towards a Formal Semantics-Based Technique for Interprocedural Slicing ${ }^{\star}$
}

\author{
Irina Măriuca Asăvoae ${ }^{1}$, Mihail Asăvoae ${ }^{1}$, and Adrián Riesco ${ }^{2}$ \\ 1 VERIMAG/UJF, France \\ \{mariuca.asavoae, mihail.asavoae\}@imag.fr \\ ${ }^{2}$ Universidad Complutense de Madrid, Spain \\ ariesco@fi.ucm.es
}

\begin{abstract}
Interprocedural slicing is a technique applied on programs with procedures which relies on how the information is passed at procedure call/return sites. Such a technique computes program slices (i.e. program fragments restricted w.r.t. a given criterion). The existing approaches to interprocedural slicing exploit the particularities of the underlying language semantics in order to compute program slices. In this paper we propose a generic technique for interprocedural slicing. More specifically, our approach works with inferred particularities of a language semantics, given as a rewriting-logic specification, and computes program slices using a term slicing-based algorithm.
\end{abstract}

Keywords: slicing, semantics, Maude, debugging.

\section{Introduction}

Complex software systems are built in a modular fashion, where modularity is implemented with functions and modules, in declarative-style programming; with classes and interfaces, in object-oriented programming; or with other means of organizing the code. Besides their structural characteristics, the modules also carry semantic information. The modules could be parameterized by types and values (e.g. the generic classes of Java and $\mathrm{C} \#$, the template classes of $\mathrm{C}++$, or the parameterized modules of Maude and OCaml) or could have specialized usability (e.g. abstract classes in object-oriented languages).

It is preferable, for efficiency reasons, that the modular characteristics of a system are preserved when new analysis techniques and tool support are developed. One possible solution to integrate both analysis and tool development is to use a formal executable framework such as rewriting logic [13. For any given program (correctly constructed w.r.t. the language syntax), the formal executable semantics, given as a rewriting logic specification, provides the set of all the concrete executions, for all the possible input data. Furthermore, the notion of a concrete execution extends to an abstract execution - as an execution with an analysis

\footnotetext{
* This research has been partially supported by MICINN Spanish project StrongSoft (TIN2012-39391-C04-04).

E. Albert and E. Sekerinski (Eds.): IFM 2014, LNCS 8739, pp. 291 306 2014

(C) Springer International Publishing Switzerland 2014
} 
tool - and we have the set of concrete executions as the basis of any abstraction (and implicitly abstract execution) of a program. One particular abstraction is program slicing [26, which computes safe program fragments (also called slices) w.r.t. a specified set of variables. A complex variant of program slicing, called interprocedural slicing, preserves the modularity of the underlying program and exploits how the program data is passed between these modules.

Interprocedural slicing is the slicing method applied on programs with procedures where the slice is computed for the entire program, taking into account the procedure calls/returns. The main problem that arises in interprocedural slicing is related to the fact that the procedure calls/returns may be analyzed with a too coarse abstraction. Namely, the abstraction relies only on the call graph without taking into account the context changes (i.e., the instantiation of the local variables during a procedure execution) occurring during a procedure call/return. Since we develop a generic, formal semantics-based slicing method, we assume that we do not know which language constructs produce these context changes. Hence, we include in our slicing method a phase for inferring these constructs, denoted in the following as scope-update constructs.

Now, our proposed technique for interprocedural slicing has two phases which could be described as follows: Given a programming language semantics $\mathcal{S}$, in the first phase we extract scope-update constructs $\mathfrak{c}$ from $\mathcal{S}$ and, in the second phase, we use these constructs for the interprocedural slicing of $\mathcal{S}$-programs (i.e., programs written in the language specified by $\mathcal{S}$ which, in fact, are well-formed terms in $\mathcal{S}$ ). In this paper we focus on the second phase of the interprocedural program slicing, meaning the term slicing-based algorithm. The first phase, (i.e. the extraction of the scope-update constructs) follows a similar meta-analysis of the language semantics as in [17, where side-effect constructs are extracted. We require $\mathcal{S}$ to be expressed as a rewriting logic theory 13, which is executable and benefits of tool support via the Maude system [3], an implementation of the rewriting logic framework. The technique to obtain the scope-update constructs is, in fact, a meta-analysis of the programming language. The interprocedural program slicing uses $\mathfrak{c}$ to collect and propagate abstract information according to the scope switches from $\mathfrak{c}$. This technique is concretised with an implementation into a generic semantics-based slicing tool developed in Maude.

For presentation purposes, we consider a WHILE language [10] with functions and local variable declarations (which introduce variable scoping); we call this extension WhileF. Then, in order to differentiate two variables based on their scopes, we need to identify scope-update constructs at the level of the semantics. Note that the meta-analysis for scope-updates used in the present work is slightly more complicated than the one for side-effects described in [17, because scopeupdates usually work in pairs so now we need to analyze $\mathcal{S}$ targeting pairs of operators (for procedure call and return). Such pairs could be explicitly presented in the language semantics definitions (through different rewrite rules for call and return) or implicitly, as in this work (with an explicit rewrite rule for call and implicit return instruction). 
Another interesting difference consists in the fact that the second program slicing step receives as input both scope-update and side-effect information, which implies heavy changes. In this case, it is necessary to address the representability of the derived scope-update constructs w.r.t. the interprocedural program slicing. Namely, a combined representation of scope-update and side-effect constructs could consist in terms representing generic skeletons for procedure summaries (a succinct representation of the procedure behavior w.r.t. its input variables).

The rest of the paper is organized as follows: Section 2 presents related work. Section 3 introduces the basic notions of program slicing and rewriting logic, used throughout the paper, while Section 4 describes the proposed program slicing as term slicing-based algorithm. Finally, Section 5 concludes and presents some lines of future work.

\section{Related Work}

Program slicing addresses a wide range of applications, from code parallelization [23] to program testing [8], debugging [21], and analysis [1211]. Since our goal is to design and implement a semantics-based program analysis tool in a rewriting-logic environment, we relate our method to both interprocedural slicing in program analysis and in rewriting logic. With respect to the general problem of program slicing, we refer the reader to the comprehensive survey of slicing techniques, in 24].

The technique of program slicing was introduced in [26, and for a given program with procedures, it computes slices using a limited form of context information (i.e. before each procedure call). The approach resembles an on-demand procedure inlining, using a backward propagation mechanism (thus, producing backward slices). Our approach takes into consideration the context-update constructs (as extracted from the formal semantics) and produces forward slices (via term slicing on the term program). Moreover, the context-update constructs play the role of symbolic procedure summaries, as in 20/11/7/22. A procedure summary is a compact representation of the procedure behavior, parameterized by its input values, which in our proposed framework is the context-update construct. The interprocedural slicing is explicit in [11|22 and implicit in 2017, and sets the support for interprocedural program analyses.

The work in 20 uses a data-flow analysis to represent how the information is passed between procedure calls. It is applied on a restricted class of programsrestricted by a finite lattice of data values -, while the underlying program representation is a mix of control-flow and control-call graphs. In comparison, our approach considers richer context information (as in [11]), while working on a similar representation of a program (as a term). The work in [7] keeps the same working structures but addresses the main data limitation of [20]. As such, the procedure summaries are represented as sets of constraints on the input/output variables. The underlying interprocedural slicing algorithm of [7] is more refined than our approach (though not generic), just because of the richer representation of context information. We follow closely the work in [11, which introduces a 
new program representation for the interprocedural slicing. In comparison, our approach does not require the explicit context representation, but uses term matching to distinguish between different contexts.

In the rewriting logic environment, there are several approaches towards debugging [1], testing [16], and analysis [17]. The dynamic slicing technique in [1] works on execution traces of the Maude model checker. In comparison, we propose a static approach built around a formal semantics and with an emphasis on computing slices for programs and not for given traces (e.g. of model checker runs). The work in [16] presents an approach to generate test cases similar to the one presented here in the sense that both use the semantics of programming languages formally specified to extract specific information. In this case, the semantic rules are used to instantiate the state of the variables used by the given program by using narrowing; in this way, it is possible to compute the values of the variables required to traverse all the statements in the program, the so called coverage. The technique in the current paper follows our previous work on language-independent program slicing in rewriting logic environment [17. Actually, the implementation of the current work is an extension of the slicing tool we developed in [17]. Both approaches share the methodology steps: (1) the initial meta-analysis of $\mathcal{S}$ and (2) the program analysis conducted over the $\mathcal{S}$-programs. More specifically, in [17] we use the classical WHILE language augmented with side-effect constructs (assignments and read/write statements) to exemplify (1) the inference of the set of side-effect language constructs in $\mathcal{S}$, and (2) the program slicing as term rewriting.

As a semantical framework, Maude has been used to specify the semantics of several languages, such as LOTOS [25], CCS [25, Java [6], or C [4]. These works describe a methodology to represent the semantics of programming languages in Maude, led to the rewriting logic semantics project [14] and to the development of the $\mathbb{K}[19$ framework. We plan to use these semantics to perform program analysis in the future.

\section{Preliminaries}

Program slicing, as introduced in [26, is a program analysis technique which computes all the program statements that might affect the value of a variable $v$ at a program point of interest, $p$. It is a common setting to consider $p$ as the last instruction of a procedure or the entire program. Hence, without restricting the proposed methodology, here we consider slices of the entire program.

A classification of program slicing techniques identifies intraprocedural slicing when the method is applied on a procedure body and interprocedural slicing when the method is applied across procedure boundaries. The key element of a methodology for interprocedural slicing is the notion of context (i.e. the values of the function/procedure parameters). Next, we elaborate on how context-aware program slicing produces better program slices than a context-forgetful one.

Let us consider, in Fig. 1, the program from [11, written as an WhileF program term, upon which we present subtleties of interprocedural slicing. We start the slicing with the set of variables of interest $\{z\}$. 
$S C$ (i.e. a set of variables) and keeps only the parts of $B$ that are subterms starting with a side-effect effect construct (from $S E$ ) and containing variables from the slicing criterion $S C$. The result of $\$(B, S C, S E)$ is given as a term $S C:: f n\left\langle f n\left(f p^{\sharp}\right)\{f s\}\right\rangle$ where $S C$ is the data flow augmented slicing criterion, $f n \in$ FunctionName is a function identifier, and $f s$ is the slice computed for $f n$. Meanwhile $f p^{\sharp}$ is the list of $f n$ 's formal parameters $f p$ filtered by $S C$, i.e., all the formal parameters not appearing in $S C$ are abstracted to a fixed additional variable $\sharp$.

Now we give a brief explanation on how the intraprocedural slicing $\$$ works. We say that a program subterm modifies a variable $v$ if the top operator is in $S E$ and $v$ appears as a leaf in a specific part of the subterm, e.g., the variable $v$ appears in the first argument of ${ }_{-}:=$- or in Local_. When such a subterm is discovered by $\$$ for a slicing variable then the slicing criterion is updated by adding the variables producing the side-effects (e.g. all variables $v^{\prime}$ in the second argument of $\_:==_{-}$) and the data flow relations $v, v^{\prime}$. We call $f s$ a skeleton subterm of $B$ and we denote this as $f s \precsim B$.

In Fig. 3 we give the slicing method, termSlicing, which receives as input the slicing criterion $s c$, the set of program functions $\mathfrak{F}_{\mathfrak{p}}$, and the set of sideeffect and context-updates syntactic constructs, $S E$ and $C U$, respectively. The output is the set of sliced function definitions slicedFnSet together with the obtained data flow augmented slicing criterion $d f s c$. Note that $\mathfrak{F}_{\mathfrak{p}}, S E$, and $C U$ are assumed to be precomputed based on the programming language semantics specification $\mathcal{S}$. The algorithm for inferring $S E$ is given in [17, Section 4]. The algorithm for inferring $C U$ goes along the same lines as the one for $S E$ and it is based on the automatic discovery of stack structures used in $\mathcal{S}$ for defining the programming language commands. For example, in WhileF the only command inducing context-updates is Call___ ( ) instruction. In the current work we assume $C U$ given in order to focus on the interprocedural slicing as term slicing method. However, we claim that termSlicing is generic w.r.t. $\mathcal{S}$ since $\mathfrak{F}_{\mathfrak{p}}, S E$, and $C U$ can be automatically derived from $\mathcal{S}$.

termSlicing is a fixpoint iteration which applies the current data-flowaugmented slicing criterion over the function terms in order to discover new skeleton subterms of the program that comply with the slicing criterion. The protocol of each iteration step is to take each currently sliced function and slice down and up in the call graph. In other words, the intraprocedural slicing is applied on every called function (i.e. goes down in the call graph) and every calling function (i.e. goes up in the call graph).

Technically, termSlicing relies on incrementally building the program slice, stored in workingSet, and the data flow augmented slicing criterion, stored in $d f s c$. This process has two phases: the initialization of workingSet and $d f s c$ (lines 0-6) and the loop implementing the fixpoint (lines 7-39).

The initialization part computes the slicing seed for the fixpoint by independently applying the intraprocedural slicing $\$\left({ }_{-}\right.$, _ $\left._{-}\right)$with the slicing criterion $s c$ for each function in the program $\mathfrak{p}$. The notation $A \cup=B$ (line 3) stands for " $A$ becomes $A \cup B$ " where $\cup$ is the set union. Similarly, $A \uplus=B$ (line 4) 


\section{termSlicing}

Input: $s c, \mathfrak{F}_{\mathfrak{p}}, S E, C U$

Output: slicedFnSet, $d f s c$

0 workingSet ${ }^{\prime}:=\emptyset ; d f s c:=\emptyset$;

1 for all $f n(\operatorname{args})\{f n B o d y\} \in \mathfrak{F}_{\mathfrak{p}}$ do

SCinit $::$ fn $\langle$ fnInitSlice $\rangle:=\$($ fnBody $,\{x \in s c \mid x \preccurlyeq f s$ or $x \preccurlyeq$ args $\}, S E)$;

workingSet ${ }^{\prime} \cup=\{$ SCinit :: fn $\langle$ fnInitSlice $\rangle\}$;

dfsc $\uplus=$ SCinit;

od

workingSet $:=\emptyset$;

while workingSet $\neq$ workingSet ${ }^{\prime}$ do

workingSet $:=$ workingSet $^{\prime}$;

for all $S C:: f n\langle$ fnSlice $\rangle \in$ workingSet do

wsFn Called $:=\emptyset$;

for all Call $\in C U$ for all Call fnCalled $\preccurlyeq f n$ Slice do

$f n C l d S C:=S C^{f n}\left\lfloor_{\text {fnCalled }}\right.$;

for all fnCldSCPrev :: fnCalled $\left\langle\left\langle_{-}\right\rangle \in\right.$ workingSet do

if $f n C l d S C \sqsubseteq f n C l d S C P r e v$ then break;

$f_{n} C l d B d:=\operatorname{getFnBody}\left(f n C a l l e d, \mathfrak{F}_{\mathfrak{p}}\right)$;

fnCldSCNew :: fnCalled $\langle$ fnCldSlice $\rangle:=\$($ fnCldBd, fnCldSC, SE $)$;

wsFnCalled $\cup=\{$ fnCldSCNew :: fnCalled $\langle$ fnCldSlice $\rangle\}$;

$S C \uplus=$ fnCldSCNew fnCalled $\rfloor^{f n}$;

od

wsFnCalling $:=\emptyset$;

for all Call $\in C U$ for all $f n$ Calling $\in \mathfrak{F}_{\mathfrak{p}}$ s.t. Call $f n \preccurlyeq f n$ Calling do fnClgSC:=SC ${ }_{f n} \Gamma^{\text {fnCalling }}$;

for all fnCallingSCPrev :: fnCalling $\langle-\rangle \in$ workingSet do

if $f_{n} C l g S C \sqsupseteq f n C a l l i n g S C P r e v$ then break;

$f n C l g B d:=\operatorname{getFnBody}\left(\right.$ fnCalling, $\left.\mathfrak{F}_{\mathfrak{p}}\right)$;

fnClgSCNew :: fnCalling $\langle$ fnClgSlice $\rangle:=\$($ fnClgBd, fnClgSC,SE);

wsFnCalling $\cup=\{$ fnClgSCNew :: fnCalling $\langle$ fnClgSlice $\rangle\}$;

$S C \uplus=$ fnClgSCNew $\left.^{\text {fnCalling }}\right\rceil_{f n} ;$

od

$f n B d:=\operatorname{getFnBody}\left(f n, \mathfrak{F}_{\mathfrak{p}}\right)$;

SCNew :: fn $\langle$ fnSliceNew $\rangle:=\$(f n B d, S C, S E)$;

dfsc $\uplus=S C N e w$;

for all Call $\in C U$ for all Call fnCalled $\preccurlyeq f n S l i c e N e w$ do

if _ :: fnCalled $\left\langle \__{-}\{\}\right\rangle \in w$ SetFnCalled then

od

fnSliceNew := erraseSubterm(Call fnCalled, fnSliceNew)

workingSet ${ }^{\prime} \amalg=\{S C N e w:: f n\langle$ fnSliceNew $\rangle\}$ ש wsFnCalled $\amalg$ wsFnCalling;

od

39 od

40 slicedFnSet $:=\operatorname{get}\langle\rangle \operatorname{Content}($ workingSet $)$

Fig. 3. Program slicing as term slicing algorithm

is the union of two data dependency graphs. Namely, $A \uplus B$ is the set union for graph edges filtered by the criterion that if a variable $v$ is independent in $A$ but dependent in $B$ (i.e. there exists an edge $\mathscr{-}$ - with $v$ on one of the 
ends) then the independent variable $v$ is eliminated from $A \uplus B$. For example, the initialization step applied on the program in Fig. 1 produces the following workingSet': z :: $\operatorname{Inc}\langle\operatorname{Inc}(\mathbf{z})\{\operatorname{Call} \operatorname{Add}(\mathbf{z}, \sharp)\}\rangle, \emptyset:: \operatorname{Main}\langle\operatorname{Main}()\{\}\rangle, \emptyset::$ $\mathrm{A}\langle\mathrm{A}(\sharp, \sharp)\{\}\rangle, \emptyset:: \operatorname{Add}\langle\operatorname{Add}(\sharp, \sharp)\{\}\rangle$.

The fixpoint loop (lines 7-39) discovers the call graph in an on-demand fashion using the context-update set $C U$, which directs the fixpoint iteration towards applying the slicing on the called/calling function. As such, when a contextupdate (e.g. Call_(_) in the semantics of WhileF) is encountered in the current slice, we proceed to slice the called function (lines 10-19). Next, when a contextupdate of the currently considered functions is encountered, we proceed again to slice the calling function (lines 20-29). Each time we update the current dataflow-augmented slicing criterion and the slice of the current function (lines 3036). For example, the discovery of the call graph starting with the function $\operatorname{Inc}(\mathbf{z})\{\operatorname{Call} \operatorname{Add}(\mathbf{z}, \sharp)\}$ in the program from Fig. 1 adds, during the first iteration of the fixpoint-loop, the called function $\operatorname{Add}(a, b)\{a:=a+b\}$ and the calling function $\mathrm{A}(\sharp, \mathrm{y})\{\mathrm{Call} \operatorname{Inc}(\mathrm{y})\}$. We iterate this process until the skeleton subterm of every function is reached, i.e., workingSet is stable, e.g., see the result from Fig. 2. Note that the stability of workingSet induces the stability of $d f s c$, the data flow augmented slicing criterion.

We now describe in more details each of the three parts of the fixpoint loop: the called (lines 10-19), the calling (lines 20-29), and the current (lines 30-36) functions. The called and calling parts have a similar flow with slight differences in the operators used. They can be summarized as:

$$
\begin{gathered}
S C \uplus=S C^{f n}\left\lfloor_{\text {fnCalled }} \text { filtered } \$(\text { fnCalled }, \sqsubseteq){ }_{\text {fnCalled }}\right\rfloor^{f n} \\
S C \uplus=S C_{f n}\left\lceil^{\text {fnCalling }} \text { filtered } \$(\text { fnCalling }, \sqsupseteq)^{\text {fnCalling }}\right\rceil_{f n}
\end{gathered}
$$

where $f n$ is the name of the current function, fnCalled is the name of a functions called from $f n$, and $f n$ Calling is the name of a function which is calling $f n$.

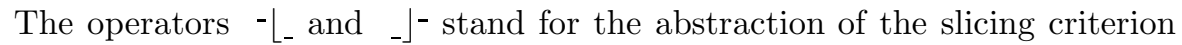
downwards in the calling graph from $f n$ into $f n$ Called and back, respectively. The abstraction ${ }^{f n}\left\lfloor_{\text {fnCalled }}\right.$ pivots on the actual parameters of fnCalled and, based on patterns of function calls, it maps the actual parameters of fnCalled from the current environment $S C:: \mathrm{fn}$ into the environment of fnCalled. The abstraction fnCalled $\rfloor^{f n}$ renders the reverse mapping from the (sliced) called environment back into the current one. Similarly for the _- - and - $\rceil_{-}$operators, which perform the abstraction upwards in the call graph from fn to fnCalling, pivoting on the parameters of $f n$. For example, for program Px from Fig. 1 we

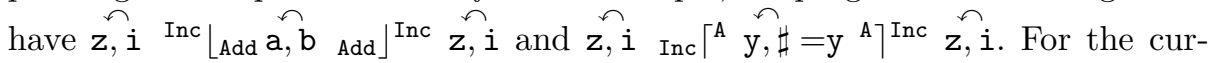
rent work, the only pattern of function calls that we have experimented is the complete list of call-by-reference parameters.

The operator filtered $\$(f n C$, rel) (lines $13-17$ and $23-27)$ is a filtered slicing of $f n C$, where the filter is a relation between the current abstraction of $S C$ and previously computed slicing criterions for the called/calling function $f n C$. We say that $S C \sqsubseteq S C P r e v$ if $S C$ is a subgraph of SCPrev such that there is no edge 
$\stackrel{\curvearrowleft}{v, v^{\prime}}$ in SCPrev where $v$ is a node in $S C$ and $v^{\prime}$ is a function parameter which is not in $S C$. This means that $S C$ has no additional dependent data $v^{\prime}$ among the function parameters that should participate to the current slicing criterion. For example, this relation is exploited for the call of function Add in function A from Fig. 1. Namely, the algorithm discovers the relation a, b for Add's parameters upon the call of Add from function Inc. Later, when function Add is called in A only with parameter $b$, the subgraph relation $\mathrm{b} \sqsubseteq \mathrm{a}, \mathrm{b}$ shows that the already sliced $\operatorname{Add}(a, b)$ contains the slice of $\operatorname{Add}(\sharp, b)$ hence, there is nothing else to be done for this function. Meanwhile, $S C \sqsupseteq S C P r e v$ is defined as SCPrev $\sqsubseteq S C$ due to the fact that now the sense in the dependency graph is reversed and so the slicing criterion in the calling function (SCPrev) is the one to drive the reasoning. Hence, if the filter relation is true then the new slice is not computed anymore (lines 14 and 24) because the current slicing criterion is subsumed by the previous computation.

In lines 30-36 we compute a new slice for the current function $f n$ and in line 37 we collect the slices currently computed for the program functions.

Lines 30-36 are more of a beautification of the slice of the currently sliced function $f n$. This beautification is made by the elimination from the slice of any context-update subterm Call fnCalled having an empty body for the currently computed slice (lines 33-36). For example, the call to function Add from function A, i.e., $\operatorname{CallAdd}(\sharp, b)$, is eliminated from the slice computed for function A due to the emptiness of the sliced body of function Add starting with the slicing criterion $b$. Note that this fact can be concluded only from the data-flow relation among the parameters of a function, provided that we add a special symbol $\ell$ for the local variables such that any function parameter $v$ depending on some local variable is going to appear as connected to $\ell$, i.e., either $\hat{v, \ell}$ or $\hat{\ell}, v$. Namely, in function Inc from Fig. 1 1 we have $\mathbf{z}, \ell$ due to the fact that Add brings $\tilde{z}, \mathbf{i}$ in the $S C$ of Inc. However, in what follows we do not insist on the data dependency on local variables in order not to burden the notation.

Finally, in line 37 we collect all the slices computed at the current iteration in workingSet' . Note that _ _ _ operator from line 37 is an abstract union which first computes the equivalence class of slices for each function, based on the graph inclusion of the data-flow-augmented slicing criterion, and then performs the union of the results. Namely, if there is a function with $F$ with three parameters $x, y, z$ such that $\stackrel{\curvearrowleft}{x, y}$ and $z$ is independent, and if at some iteration of the fixpoint we have the slice $F(x, y, \sharp)\left\{B_{x, y, \sharp}\right\}$ in the set wsFnCalled and $F(\sharp, \sharp, z)\left\{B_{\sharp, \sharp, z}\right\}$ in the set wsFnCalling, then in workingSet' we have $F x, y, z\left\{B_{x, y, z}\right\}$ where in $B_{x, y, z}$ we put together the two skeletons $B_{x, y, \sharp}$ and $B_{\sharp, \sharp, z}$.

Recall that, in Section 3, we described two interprocedural slicing methods presented in [26] and [11, being the second one more precise than the first one. In our approach the difference is based solely on the data flow relation we use for $\$$. Hence, we can distinguish two types of termSlicing: the naïve one where the data flow relations are ignored and the savvy one which collects and uses data flow relations. Note that the data flow relation is currently assumed as given. 


\begin{tabular}{|c|c|c|}
\hline $\begin{array}{c}\text { Slicing } \\
\text { variables }\end{array}$ & $\begin{array}{l}\text { Function } \\
\text { contexts }\end{array}$ & $\begin{array}{c}\text { Computed slice } \\
\text { (identified subterms) }\end{array}$ \\
\hline $\bar{z}:: \mathrm{\top}$ & $\begin{array}{c}{ }^{\top}\left\lfloor\text { Inc } \mathbf{z} \rightarrow \mathbf{z}_{\text {Inc }}\right\rfloor^{\top} \\
{ }^{\top}\left\lfloor\text { Main } \sharp=\emptyset_{\text {Main }}\right\rfloor^{\top}, \ldots\end{array}$ & $\begin{array}{c}\operatorname{Inc}(\mathbf{z})\{\operatorname{Call} \operatorname{Add}(\mathbf{z}, \sharp)\} \\
\operatorname{Main}()\{\}, \mathrm{A}(\sharp, \sharp)\{\}, \mathrm{B}(\sharp, \sharp)\{\}, \operatorname{Add}(\sharp, \sharp)\{\}\end{array}$ \\
\hline \begin{tabular}{|c|}
$\mathrm{z}:: \operatorname{Inc}$ \\
$\curvearrowleft$ \\
$\mathrm{z}, \mathrm{i}:: \operatorname{Inc}$ \\
$\curvearrowleft$ \\
$\mathrm{z}, \mathrm{i}:: \operatorname{Inc}$ \\
$\bumpeq$ \\
$\mathrm{z}, \mathrm{i}:: \operatorname{Inc}$ \\
\end{tabular} & 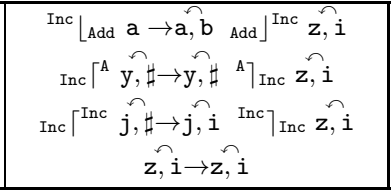 & $\begin{array}{c}\operatorname{Add}(\mathrm{a}, \mathrm{b})\{\mathrm{a}:=\mathrm{a}+\mathrm{b}\} \\
\mathrm{A}(\sharp, \mathrm{y})\{\operatorname{Call} \operatorname{Inc}(\mathrm{y})\} \\
\operatorname{Inc}(\sharp)\{\operatorname{Local} \mathbf{i}, \mathbf{j} ; \mathbf{i}:=1 ; j:=\mathrm{i} ; \operatorname{Call} \operatorname{Add}(\sharp, \mathbf{i}) ; \operatorname{Call} \operatorname{Inc}(\mathrm{j})\} \\
\operatorname{Inc}(\mathbf{z})\{\operatorname{Local} \mathrm{i} ; \mathrm{i}:=1 ; \operatorname{Call} \operatorname{Add}(\mathbf{z}, \mathbf{i})\}\end{array}$ \\
\hline $\begin{array}{l}\mathrm{y}:: A \\
\mathrm{y}:: A \\
\mathrm{y}:: A \\
\mathrm{y}:: A\end{array}$ & 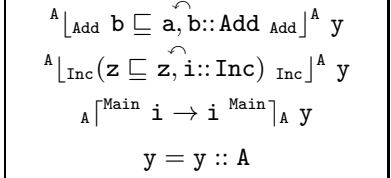 & $\begin{array}{c}\square \\
\square \\
\operatorname{Main}()\{\text { Local } i ; i=1 ; \text { While } i<1 \text { Do Call A( } \sharp, i)\} \\
\square\end{array}$ \\
\hline \begin{tabular}{|l|}
$\mathrm{a}, \mathrm{b}::$ Add \\
$\curvearrowleft$ \\
$\mathrm{a}, \mathrm{b}::$ Add \\
$\curvearrowleft \curvearrowleft$ \\
$\mathrm{a}, \mathrm{b}::$ Add \\
\end{tabular} & 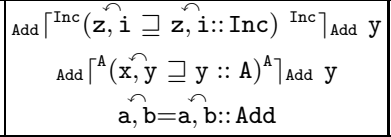 & $\begin{array}{l}\square \\
\square \\
\square\end{array}$ \\
\hline 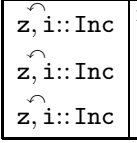 & 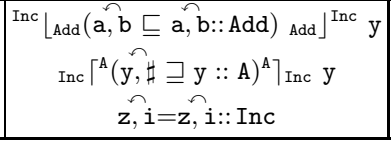 & $\begin{array}{l}\square \\
\square \\
\square\end{array}$ \\
\hline $\begin{array}{l}\mathrm{y}:: \mathrm{A} \\
\mathrm{y}:: \mathrm{A} \\
\mathrm{y}:: \mathrm{A} \\
\mathrm{y}:: \mathrm{A}\end{array}$ & 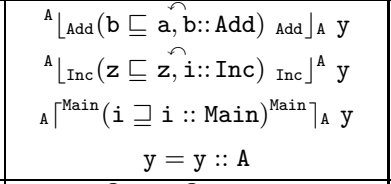 & $\begin{array}{l}\square \\
\square \\
\square \\
\square\end{array}$ \\
\hline \begin{tabular}{|l|}
$\mathrm{a}, \mathrm{b}::$ Add \\
$\curvearrowleft$ \\
$\mathrm{a}, \mathrm{b}::$ Add \\
$\curvearrowleft \curvearrowleft$ \\
$\mathrm{a}, \mathrm{b}::$ Add
\end{tabular} & 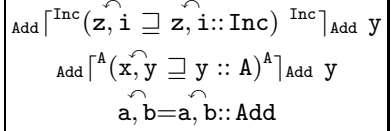 & $\begin{array}{l}\square \\
\square \\
\square\end{array}$ \\
\hline \begin{tabular}{|c|}
$z, i:: \operatorname{Inc}$ \\
$\curvearrowleft$ \\
$z, i:: \operatorname{Inc}$ \\
$\approx, i:: \operatorname{Inc}$
\end{tabular} & 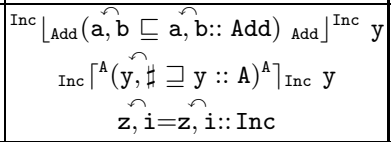 & $\begin{array}{l}\square \\
\square \\
\square\end{array}$ \\
\hline 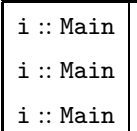 & $\begin{array}{c}{ }^{\text {Main }}\left\lfloor_{A}(y \sqsubseteq y:: A)_{A}\right\rfloor^{\text {Main }} i \\
\left.{ }_{B}(\# \sqsubseteq \emptyset:: B)_{B}\right\rfloor^{\text {Main }} i \\
i=i:: \text { Main }\end{array}$ & $\begin{array}{l}\square \\
\square \\
\square\end{array}$ \\
\hline
\end{tabular}

Fig. 5. Program slicing as term slicing - the fixpoint iterations

termSlicing terminates because there exists a finite set of function skeleton subterms, a finite set of data flow graphs, a finite set of edges in the call graph for each function, and any loop in the call graph is solved based on the data flow graph ordering. Moreover, termSlicing produces a valid slice because it exhaustively saturates the slicing criterion. However, the obtained slice is not minimal due to the skeletons union $\mathbb{U}$. Still, there is a consistent difference between the naïve and the savvy methods. In order to achieve a better degree of minimality we have to apply abstractions on the data-flow-augmented slicing criterion. 


\subsection{System Description}

We briefly present in this section our prototype which is implemented in Maude 3. The source code is available at http://maude.sip.ucm.es/slicing/ A key distinguishing feature of Maude is its systematic and efficient use of reflection (i.e. Maude's capability of handling and reasoning about terms that represent specifications described in Maude itself) through its predefined META-LEVEL module [3, Chapter 14]. We have used these features to implement a tool that receives a set of definitions, a sort where the computations take place, and a set of slicing variables. Since all these elements can be used as usual data, we can traverse the semantic rules, analyze them, and execute the program using them. Note that the user has to provide the rules responsible for context-update while the

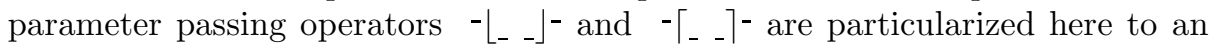
all-parameters-ordered-pass-by-reference pattern.

The tool is started by loading into Maude the slicing.maude file available at the webpage above. It starts an input/output loop where modules and commands can be introduced by enclosing them in parentheses. Once the module with the semantics has been loaded, we have to introduce ESt, the sort for the mapping between variables and values, and RWBUF, the sort for the read/write buffer, as the sorts responsible for the side effects. Similarly, we indicate that CallF is the rule for context-update:

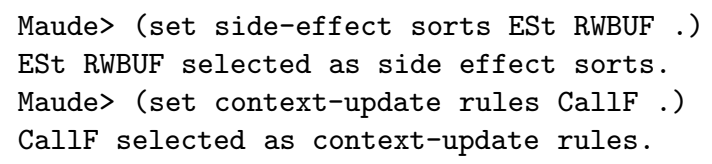

We can now start the slicing process by indicating that Statement is the sort for instructions, myFuns is a constant standing for the definition of the functions Main, A, Add, and Inc from Figure 4, and $\mathrm{z}$ is the slicing variable. The tool displays the relevant variables and the sliced code for each function as:

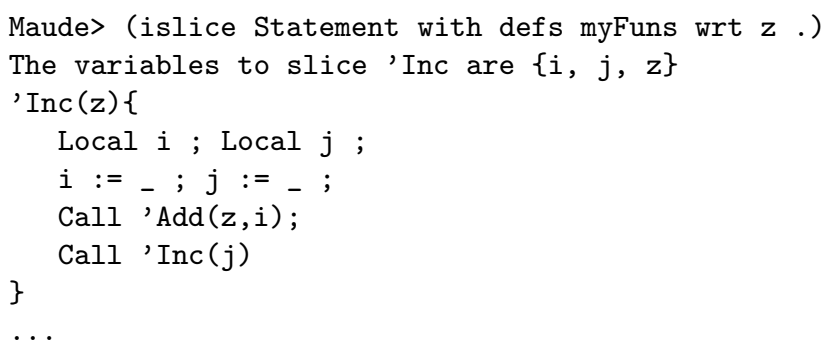

We test our proposed method for interprocedural slicing on a set of benchmarks addressing embedded and real-time applications. As such, we use a set of small examples, grouped under the name bundle, from a survey 24] on program slicing techniques, automatically-generated code from typical Scade designs [5], as well as a standard set of real-time benchmarks - called PapaBench [15. In Figure 6, each program is identified by name, a short description, size parameters (LOC, number of functions, \#funs, and function calls, \#calls), and the 


\begin{tabular}{|c|c|c|c|c|c|}
\hline Name & Program Description & LOC & \#funs & \#calls & red (\%) \\
\hline \hline bundle & $\begin{array}{c}\text { A collection of (extended) } \\
\text { examples from [24] }\end{array}$ & 71 & 7 & 25 & $38 \%$ \\
\hline \hline selector_2 & $\begin{array}{c}\text { Generated code from } \\
\text { SCADE design - 2 SSM }\end{array}$ & 426 & 6 & 11 & $91 \%$ \\
\hline \hline selector_3 & $\begin{array}{c}\text { Generated code from } \\
\text { SCADE design - 3 SSM }\end{array}$ & 455 & 7 & 19 & $85 \%$ \\
\hline \hline autopilot & PapaBench - autopilot & 1384 & 95 & 214 & $74 \%$ \\
\hline \hline fbw & PapaBench - fly_by_wire & 638 & 41 & 110 & $78 \%$ \\
\hline
\end{tabular}

Fig. 6. Set of benchmark programs for interprocedural slicing

average reduction in the number of statements, for several runs with different sets of slicing variables. This reduction shows that the methodology works better on bigger programs (the bundle, with very small examples, presents the lowest reduction, because all variables are closely related). The Scade benchmarks, explained below, present the greatest reduction because the variables have very specific behaviors, hence allowing a very efficient use of slicing.

The Scade Suite development platform [5] is a mixed synchronous language, combining variants of Lustre [9] (i.e. data-flow) and Esterel [2] (i.e. control-flow). Scade facilitates the design of embedded and real-time systems in a modular fashion, and the modularity is preserved in the generated C code. The two Scade designs - selector_2 and selector_3 - consist of two, and respectively three, parallel state machines (called SSM - Safe State Machines) which embed in their states calls to external functions and constrain (via shared variables) how these state machines communicate among them.

PapaBench is extracted from an actual real-time system for Unmanned Aerial Vehicle (UAV) and consists of two programs fly_by_wire and autopilot, designed to run on different processors. The application consists of a number of tasks which are executed in a control loop. For example, the autopilot program focuses on the UAV airframe and has eight different tasks (e.g. for controlling the navigation, stabilisation, altitude or communication - radio or GPS).

We test our interprocedural slicing at the level of the entire program as well as at the level of each task. Let us consider the function radio_control_task (in autopilot) which manages radio orders based on various operation modes (e.g. PITCH, ROLL, THROTTLE, etc) and sets new values for several flight parameters (e.g. desired_roll or desired_pitch). This particular function has a call graph of about 21 nodes. We could use, for example, a slicing criterion which consists of all program variables used in radio_control_task in order to investigate the tasks which are depending (i.e. their intraprocedural slice is not empty) or not on the computation of radio_control_task. The interprocedural slice shows a dependence of the radio_control_task with tasks such as 
altitude_control_task and climb_control_task, which rely on global flight parameters used by the radio controller. This testing strategy is applied on all benchmarks and, together with the resulting traces and the Scade designs, are available on the tool webpage at http://maude.sip.ucm.es/slicing/.

\section{Concluding Remarks and Ongoing Work}

The formal language definitions based on the rewriting logic framework support program executability and create the premises for further development of program analyzers. In this paper we have presented a generic algorithm for interprocedural slicing based on results of meta-level analysis of the language semantics. In summary, the slicing prerequisites are: side-effect and context-update language constructs with data flow information for the side-effect constructs and parameter passing patterns for the context-update constructs. The actual program slicing computation, presented in the current work, is done through term slicing and is meant to set the aforementioned set of prerequisites. This work complements the recent advances in semantics-constructed tools for debugging [18, automated testing [16], and program analysis 17.

From the prototype point of view, we also plan to investigate the automatic inference of the newly identified slicing prerequisites, i.e., meta-analysis for context-updates deduction and parameter passing pattern inference. This would greatly simplify the user task, since he will just introduce the program and the slicing criterion and the tool would be in charge of computing all the required constructors. We also have to further develop the already existing side-effect extraction with data flow information. Finally, we aim to develop the method for language semantics defined in Maude but also in $\mathbb{K}[19$.

\section{References}

1. Alpuente, M., Ballis, D., Espert, J., Romero, D.: Backward trace slicing for rewriting logic theories. In: Bjørner, N., Sofronie-Stokkermans, V. (eds.) CADE 2011. LNCS, vol. 6803, pp. 34-48. Springer, Heidelberg (2011)

2. Berry, G., Gonthier, G.: The esterel synchronous programming language: Design, semantics, implementation. Sci. Comput. Program (SCP) 19(2), 87-152 (1992)

3. Clavel, M., Durán, F., Eker, S., Lincoln, P., Martí-Oliet, N., Meseguer, J., Talcott, C.: All About Maude - A High-Performance Logical Framework. LNCS, vol. 4350. Springer, Heidelberg (2007)

4. Ellison, C., Rosu, G.: An executable formal semantics of c with applications. In: POPL, pp. 533-544 (2012)

5. Esterel Technologies Scade Language Reference Manual 2011 (2011)

6. Farzan, A., Chen, F., Meseguer, J., Roşu, G.: Formal analysis of java programs in javaFAN. In: Alur, R., Peled, D.A. (eds.) CAV 2004. LNCS, vol. 3114, pp. 501-505. Springer, Heidelberg (2004)

7. Gulwani, S., Tiwari, A.: Computing procedure summaries for interprocedural analysis. In: De Nicola, R. (ed.) ESOP 2007. LNCS, vol. 4421, pp. 253-267. Springer, Heidelberg (2007) 
8. Harman, M., Danicic, S.: Using program slicing to simplify testing. Journal of Software Testing, Verification and Reliability 5, 143-162 (1995)

9. Halbwachs, N., Caspi, P., Raymond, P., Pilaud, D.: The synchronous dataflow programming language lustre. In: Proc. of the IEEE, pp. 1305-1320 (1991)

10. Hennessy, M.: The Semantics of Programming Languages: An Elementary Introduction Using Structural Operational Semantics. John Wiley \& Sons (1990)

11. Horwitz, S., Reps, T., Binkley, D.: Interprocedural slicing using dependence graphs. In: Conference on Programming Language Design and Implementation, PLDI 1988, pp. 35-46 (1988)

12. Jhala, R., Majumdar, R.: Path slicing. In: Proc. of the 2005 ACM SIGPLAN Conference on Programming Language Design and Implementation, PLDI 2005, pp. 38-47. ACM Press (2005)

13. Martí-Oliet, N., Meseguer, J.: Rewriting logic: roadmap and bibliography. Theor. Comput. Sci. 285(2), 121-154 (2002)

14. Meseguer, J., Roşu, G.: The rewriting logic semantics project. Theoretical Computer Science 373(3), 213-237 (2007)

15. Nemer, F., Cassé, H., Sainrat, P., Bahsoun, J.P., De Michiel, M.: PapaBench: a Free Real-Time Benchmark. In: WCET 2006 (2006)

16. Riesco, A.: Using semantics specified in Maude to generate test cases. In: Roychoudhury, A., D’Souza, M. (eds.) ICTAC 2012. LNCS, vol. 7521, pp. 90-104. Springer, Heidelberg (2012)

17. Riesco, A., Asăvoae, I.M., Asăvoae, M.: A generic program slicing technique based on language definitions. In: Martí-Oliet, N., Palomino, M. (eds.) WADT 2012. LNCS, vol. 7841, pp. 248-264. Springer, Heidelberg (2013)

18. Riesco, A., Verdejo, A., Martí-Oliet, N., Caballero, R.: Declarative debugging of rewriting logic specifications. Journal of Logic and Algebraic Programming (2012)

19. Şerbănuţă, T., Ştefănescu, G., Roşu, G.: Defining and executing P systems with structured data in K. In: Corne, D.W., Frisco, P., Păun, G., Rozenberg, G., Salomaa, A. (eds.) WMC 2008. LNCS, vol. 5391, pp. 374-393. Springer, Heidelberg (2009)

20. Sharir, M., Pnueli, A.: Two approaches to interprocedural data flow analysis. In: Program Flow Analysis, pp. 189-233 (1981)

21. Silva, J., Chitil, O.: Combining algorithmic debugging and program slicing. In: PPDP, pp. 157-166. ACM Press (2006)

22. Sridharan, M., Fink, S.J., Bodík, R.: Thin slicing. In: PLDI, pp. 112-122 (2007)

23. Tian, C., Feng, M., Gupta, R.: Speculative parallelization using state separation and multiple value prediction. In: Proc. of the 2010 International Symposium on Memory Management, ISMM 2010, pp. 63-72. ACM Press (2010)

24. Tip, F.: A survey of program slicing techniques. J. Prog. Lang. 3(3) (1995)

25. Verdejo, A., Martí-Oliet, N.: Executable structural operational semantics in Maude. Journal of Logic and Algebraic Programming 67, 226-293 (2006)

26. Weiser, M.: Program slicing. In: Proc. of the 5th International Conference on Software Engineering, ICSE 1981, pp. 439-449. IEEE Press (1981) 